\title{
The effects of evaluation, activity, and potency on frequency estimates
}

\author{
MARGARET W. MATLIN and MICHAEL R. STONE \\ State University of New York, College at Geneseo, Geneseo, New York 14454
}

\begin{abstract}
This study examined the influence of semantic factors on frequency estimates for English words. Words varying in either evaluation, activity, or potency appeared on a sheet of paper $0,1,4$, or 8 times. Subjects estimated how many times they had seen each individual word and then estimated the total number of times they had seen words which were high, medium, or low on the relevant semantic dimensions. Results showed that: (1) Individual word estimates are significantly higher for words that are positive (word frequency of 8 only); (2) Individual word estimates are significantly higher for words that are active and soft; (3) Total word estimates are significantly higher for words that are active.
\end{abstract}

Previous research has indicated that subjects give higher frequency estimates for positive stimuli than for negative stimuli. Stang (Note 1) demonstrated this effect using schematic faces bearing smiling, neutral, and negative expressions. Matlin and Stang (in press) confirmed these earlier results using nonsense word stimuli which varied in evaluative ratings. Thus, when frequency of exposure is manipulated in the laboratory, subjects believe that positive stimuli appeared more often than negative stimuli.

To our knowledge, the only investigation of frequency estimates for English words varying in evaluative ratings involves subjects estimating the natural frequency of occurrence for positive, neutral, and negative words in written English (Matlin \& Stang, Note 2). In that study, frequency estimates were higher for positive and negative words than for normal words.

The present study, in which frequency is manipulated in the laboratory, is concerned with frequency estimates for English words rated "positive," "neutral," and "negative" on the Jenkins norms (Jenkins, Russell, \& Suci, 1958). It also examines the effects of two other semantic factors, activity and potency, upon frequency estimates.

\section{METHOD}

\section{Subjects}

Subjects were 115 students (106 female, 9 males) from the introductory psychology pool. They were tested in a single large group, which the 12 experimental conditions randomly determined by which stimulus sheet they received.

\section{Design}

Frequency was varied within subjects. Each subject saw two stimuli in each of four frequency categories: $8,4,1$, and 0 . The design of the experiment provided for two between-subjects variables: three semantic factors (evaluation, potency, and activity) and four different lists, which counterbalanced for frequency.

\section{Stimulus Material}

We derived one list of 24 English words for each of the three semantic factors, evaluation, potency, and activity. We constructed the lists in the following manner, exemplified by the evaluation word list. Initial lists were constructed by isolating all words designated positive (1.00-2.50), neutral (3.50-4.50), and negative (5.50-7.00) on Scale 11 from the Jenkins norms. All words were eliminated which were polarized on the other two semantic factors; i.e., words were retained only if they showed ratings between 2.50 and 5.50 on the other two scales, activity (Scale 5) and potency (Scale 8).

The final list, consisting of 8 positive, 8 neutral, and 8 negative words, was selected by balancing for Kucera-Francis frequency (Kucera \& Francis, 1967), number of syllables, and part of speech. Analogous procedures were followed in constructing the potency and the activity lists.

Each subject saw the display of stimuli on $8 \frac{1}{2} \times 11$ in. photocopy of a typed page. The stimuli were arranged in a matrix 6 columns wide with 13 words in each column; rows were double spaced. The frequency estimation sheet consisted of a single sheet of paper listing the 24 experimental stimuli, preceded by a blank space. Each sheet was folded and inserted in a business envelope. The stimulus envelope was labeled I and the frequency estimation envelope was labeled II. The two envelopes were stapled together so that each subject would receive a single packet.

\section{Procedure}

The experimenter distributed one pack of envelopes to each subject. She then instructed them to open Envelope I and look very carefully at the list of English words. The subjects examined the list for $4 \mathrm{~min}$. and then returned the stimulus sheet to its envelope. Then they opened Envelope II, containing the frequency estimation sheet. They were instructed to guess how many times they had seen each word on the stimulu sheet, supplying a zero if they had not seen the word before.

They were instructed to guess how many times, in total, they had seen words in each of three categories. The subjects were asked to look at the code at the bottom of the page (E, A, or P) and to respond appropriately on the reverse side of the page. Subjects in the evaluative condition were supplied with the category titles "good words," "neutral words," and "bad words." Similar titles were supplied for conditions of activity ("active," "neutral," and "inactive"" and potency ("hard," "neutral," and "soft"). The experimenter then collected all envelopes.

\section{RESULTS}

Two sets of analyses were performed, one using the 


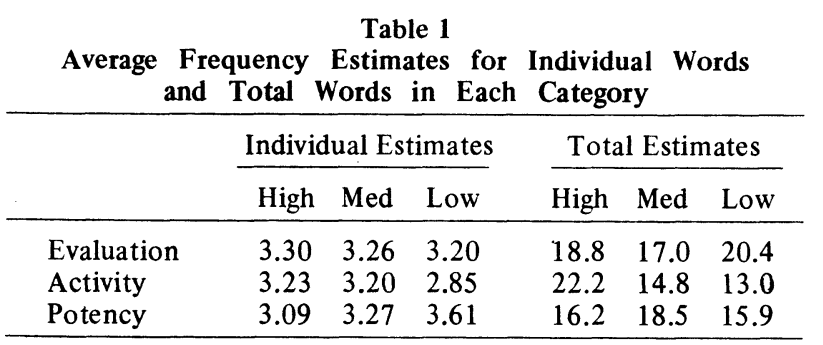

estimates given for the individual words and another using the estimates for the total number of words in each of the three categories.

\section{Estimates for Individual Words}

Three analyses of variance were conducted on these data, a separate one for each semantic dimension (see Table 1).

Evaluation. Frequency was the only significant main factor $(F=154.83, \mathrm{df}=3 / 108, \mathrm{p}<.001)$. In addition, the interaction, Evaluation by Frequency, was significant $(F=6.22, \mathrm{df}=6 / 216, \mathrm{p}<.001)$, and so was the triple interaction, Evaluation by Frequency by List $(\mathrm{F}=1.86, \mathrm{df}=18 / 216, \mathrm{p}<.05)$.

The significant factors were analyzed further. Frequency estimates increased with true frequency, as expected. A Newman-Keuls analysis of the Frequency by Evaluation interaction showed that evaluation influenced frequency estimates only when words appeared eight times. For these high frequency words, positive and neutral words were judged significantly more frequent than negative words $(\mathrm{p}<.01)$. The first two categories did not differ significantly from each other. Inspection of the triple interaction Evaluation by Frequency by List showed that for Lists 2, 3, and 4, words ween eight times had higher frequency estimates for positive words than for neutral words, which were in turn higher than for negative words. However, this pattern was reversed for List 1.

Activity. Two main factors were significant: Activity $(\mathrm{F}=3.78$, df $=2.70, \mathrm{p}<.05)$ and frequency $(\mathrm{F}=$ 106.91, $\mathrm{df}=3 / 105, \mathrm{p}<.05$ ). Also, the triple interaction List by Frequency by Activity was significant $(F=2.43$, $\mathrm{df}=18 / 210, \mathrm{p}<.01)$.

Further examination showed that frequency estimates increased with true frequency. A Newman-Keuls analysis of the activity factor showed that frequency estimates were lower for inactive words than for either active or neutral words ( $\mathrm{p}<.05$ ), but these last two categories did not differ significantly from each other. Inspection revealed no consistent pattern in the frequency estimates underlying the triple interactions.

\section{Potency}

Two main factors were significant: Potency $(\mathrm{F}=4.55$, df $=2 / 68, p<.02)$ and Frequency $(F=227.44$, df $=$ $3 / 109, p<.001)$. In addition, the interaction List by Frequency was significant $[F=3.04$, df $=9 / 102$, $\mathrm{p}<.01]$.
Frequency estimates increased with true frequency. A Newman-Keuls analysis of the potency factor showed, contrary to our expectations, that frequency estimates were significantly higher for low potency words than for medium potency words $(\mathrm{p}<.05)$ or high potency words $(p<.01)$, but the difference between these latter two groups was significant. Inspection of the List by Frequency interaction showed that subjects on one list overestimated true frequency of words which appeared 8 times, while subjects on the other three lists underestimated it.

\section{Estimates for Total Words}

Three analysis of variance were conducted on these data, a separate one for each semantic dimension (see Table 1). The analyses for evaluation and potency showed no significant main factors. The analysis for activity demonstrated that activity did influence estimates of the total number of words $(\mathrm{F}=7.98, \mathrm{df}=$ $2 / 76, \mathrm{p}<.01)$. A Newman-Keuls analysis showed that high activity words received higher total frequency estimates than either medium or low activity words $(p<.01)$. These latter two groups did not differ significantly from each other.

\section{CONCLUSION}

The three semantic dimensions influence frequency estimates. When subjects are asked to judge how many times they have seen an English word, their estimates will be higher if the word is positive (high frequency only), active, and soft. The limited influence of evaluation on frequency estimates provides a partial replication of Stang (1974) and Matlin and Stang (in press). The influence of activity on frequency estimates coincides with intuitive predictions. However, it is puzzlirg that subjects give higher frequency estimates for soft words than for hard words. Perhaps this effect is limited to our particular word sample or to a predominantly female subject population.

Some additional information is provided by subjects' estimates of the total occurrences of words in each category. Only the analysis of the activity data demonstrated a significant effect for the total estimates as well as the individual estimates. These data confirm the observation of Stang (Note 1) that individual word estimates and total word estimates do not necessarily operate in the same way.

\section{REFERENCES}

Jenkins, J. J., Russell, W. A., \& Suci, G. J. An atlas of semantic proviles for 360 words. American Journal of Psychology, $1958,71,688-699$.

Kucera, H., \& Francis, W. N. Computational analysis of present-day American English. Providence, R. I.: Brown University Press, 1967.

Matlin, M. W., \& Stang, D. J. Some determinants of word frequency estimates. Personality \& Motor Skills (in press).

\section{REFERENCE NOTES}

1. D. J. Stang, Influence of frequency and affect on exposure frequency estimates. Paper presented at the annual meeting of the Eastern Psychological Association, Philadelphia, Pennsylvania, May, 1974.

2. M. W. Matlin and D. J. Stang. The Pollyanna Principle. (In preparation). 